\title{
Hippocampal and Prefrontal Projections to the Basal Amygdala Mediate Contextual Regulation of Fear after Extinction
}

\author{
Caitlin A. Orsini, ${ }^{1}$ Jee Hyun Kim, ${ }^{1}$ Ewelina Knapska, ${ }^{3}$ and Stephen Maren ${ }^{1,2}$ \\ ${ }^{1}$ Department of Psychology and ${ }^{2}$ Neuroscience Program, University of Michigan, Ann Arbor, Michigan 48109-1043, and ${ }^{3}$ Nencki Institute of Experimental \\ Biology, Polish Academy of Sciences, 02-093 Warszawa, Poland
}

\begin{abstract}
Knowing when and where to express fear is essential to survival. Recent work in fear extinction paradigms reveals that the contextual regulation of fear involves a neural network involving the hippocampus, medial prefrontal cortex, and amygdala. The amygdaloid basal nuclei $(\mathrm{BA})$ receive convergent input from the ventral hippocampus $(\mathrm{VH})$ and prelimbic $(\mathrm{PL})$ prefrontal cortex and may integrate $\mathrm{VH}$ and $\mathrm{PL}$ input to regulate fear expression. To examine the functional organization of this neural circuit, we used cellular imaging of $\mathrm{c}$-fos expression in anatomically defined neuronal populations and circuit disconnections to identify the pathways involved in the contextual control of extinguished fear. Before behavioral testing, we infused a retrograde tracer into the amygdala to label BA-projecting neurons in VH and PL. Rats then underwent fear conditioning and extinction and were tested for their fear to the extinguished conditioned stimulus (CS) in either the extinction context or in another context; freezing behavior served as the index of conditional fear. CS presentation outside the extinction context renewed conditional freezing and was associated with significantly more c-fos expression in BA-projecting neurons in the VH and PL than that induced by CS presentation in the extinction context. We next examined whether direct or indirect projections of $\mathrm{VH}$ to BA mediate fear renewal. Interestingly, disconnections of the VH from either the BA or PL eliminated renewal. These findings suggest that convergent inputs from both the VH and PL in the BA mediate the contextual control of fear after extinction.
\end{abstract}

\section{Introduction}

In recent years, considerable interest has emerged in the extinction of learned fear insofar as it is central to several clinical interventions, including exposure therapy. During extinction, a previously conditioned stimulus (CS) is repeatedly presented without the unconditioned stimulus (US). This results in a gradual decrease in learned fear responses, such as freezing behavior (Maren, 2001). However, extinction does not erase the original fear memory; rather, it yields a new inhibitory memory that reduces fear to the CS (Quirk and Mueller, 2008). Which memory is retrieved depends on the retrieval context; fear to an extinguished CS is suppressed in the extinction context but "renews" when it is presented outside the extinction context (Bouton and Bolles, 1979). The renewal of extinguished fear presents obvious challenges for the efficacy of behavioral interventions for fear and anxiety disorders.

Recently, substantial progress has been made in understanding the neural mechanisms for the context dependence of extinc-

\footnotetext{
Received Aug. 9, 2011; revised Sept. 15, 2011; accepted 0ct. 7, 2011.

Author contributions: C.A.O. and S.M. designed research; C.A.O., J.H.K., and E.K. performed research; C.A.O. and S.M. analyzed data; C.A.O. and S.M. wrote the paper.

This work was supported by National Institute of Mental Health Grants R01MH065961 (S.M.) and F31MH091822 (C.A.0.) and the American Psychological Association (C.A.0.).

Correspondence should be addressed to Dr. Stephen Maren, Department of Psychology, University of Michigan, 530 Church Street, Ann Arbor, Ml 48109-1043. E-mail: maren@umich.edu.

J. H. Kim's present address: Florey Neuroscience Institutes, Melbourne, Victoria 3010, Australia.

DOI:10.1523/JNEUROSCI.4095-11.2011

Copyright $\odot 2011$ the authors $\quad 0270-6474 / 11 / 3117269-09 \$ 15.00 / 0$
}

tion (Maren, 2005, 2011). This work has revealed that the hippocampus, a structure critical for context processing (Fanselow, 2000), plays an important role in the contextual modulation of fear after extinction. For example, pharmacological inactivation of either the dorsal hippocampus (Corcoran and Maren, 2001) or ventral hippocampus (VH; Hobin et al., 2006) in rats eliminates the renewal of fear to an extinguished CS outside the extinction context. Interestingly, hippocampal inactivation also eliminates the contextual modulation of CS-evoked spike firing in the amygdala after extinction (Hobin et al., 2003; Maren and Hobin, 2007), suggesting that hippocampo-amygdala projections (Canteras and Swanson, 1992; Pitkanen et al., 2000) mediate the context dependence of extinction. Because the $\mathrm{VH}$ is the primary source of contextual information to the amygdala (Pitkanen et al., 2000), it is conceivable that this direct projection is necessary for the renewal of fear. Indeed, neurons within the basal amygdala (BA) that are active during renewal receive direct projections from the $\mathrm{VH}$ (Herry et al., 2008).

Another route by which the $\mathrm{VH}$ can influence $\mathrm{BA}$ activity is via the prelimbic cortex (PL). The VH has robust projections to the PL (Vertes, 2006), which in turn has reciprocal connections with the BA (Mcdonald et al., 1996; Vertes, 2004). Prelimbic lesions impair fear expression (Blum et al., 2006; Corcoran and Quirk, 2007), and microstimulation of the PL results in both freezing behavior (Vidal-Gonzalez et al., 2006) and increases in BA firing (Likhtik et al., 2005). Additionally, PL activity during fear conditioning correlates with the expression of freezing (Burgos-Robles 
et al., 2009). Last, we have found increased c-fos expression in the PL after the renewal of fear (Knapska and Maren, 2009). Hence, it is possible that the hippocampus contributes to the context dependence of extinction through either direct or indirect projections to the BA.

To explore this question, we used functional retrograde tracing to determine whether BA-projecting neurons in the $\mathrm{VH}$ and PL are differentially activated (as indexed by c-fos expression) during renewal of fear after extinction. Asymmetric lesions were then used to disconnect the $\mathrm{VH}$ from either the BA or PL to determine the necessity of each pathway in renewal (Olton et al., 1982). Collectively, our results indicate that convergent input from the $\mathrm{PL}$ and $\mathrm{VH}$ within the $\mathrm{BA}$ is required for the contextual modulation of fear after extinction.

\section{Materials and Methods}

Subjects. Subjects were male Long-Evans rats (220-224 g; Blue Spruce), obtained from a commercial supplier (Harlan). Animals were individually housed in clear plastic hanging cages and were kept on a 14/10 h light/dark cycle and had access to food and water ad libitum. Rats were handled $15-20 \mathrm{~s} / \mathrm{d}$ for $5 \mathrm{~d}$ before the start of the experiment so as to acclimate the animals to the experimenter. All experimental procedures were performed in accordance with the protocols approved by the University of Michigan Committee on the Use and Care of Animals.

Behavioral apparatus. All behavioral sessions were performed in eight identical observation chambers $(30 \times 24 \times 21 \mathrm{~cm}$; MED Associates $)$, each located in an individual sound-attenuating cabinet. The observation chambers were constructed of two aluminum sidewalls and a Plexiglas ceiling, back, and door. The floor of each chamber consisted of 19 stainless steel rods (4 $\mathrm{mm}$ in diameter) used for delivery of the footshock US. The rods were wired to a shock source and a solid-state shock scrambler (MED Associates). To deliver the acoustic CS, a speaker was mounted on one wall of each chamber. Additionally, ventilation fans and house lights were installed in each chamber to allow for the manipulation of contexts during training, extinction, exposure, and testing. We used a three-context ("ABC") renewal procedure that allows both fear and c-fos expression evoked by an extinguished CS to be assessed independent of background fear to the context (Corcoran and Maren, 2001; see procedure below). For context A (conditioning context), house lights and room lights were on, ventilation fans (65 $\mathrm{dB}$ ) were turned on, cabinet doors were left open, and the chambers were cleaned with $1 \%$ acetic acid. For context B (extinction and test context), house lights and ventilation fans were turned off, the cabinet doors were closed, and the chambers were cleaned with $1 \%$ ammonium hydroxide. Additionally, the room was illuminated by fluorescent red lights. For context C (test context), house lights were on, ventilation fans were off, the room was illuminated with fluorescent red light, and cabinet doors were left open. Black Plexiglas floors were placed on the grid of each chamber, and chambers were cleaned with $10 \%$ ethanol. In each context, stainless steel pans were filled with a thin layer of the respective odor of the context and inserted below the grid floor.

During the behavioral sessions, motor activity was measured by recording the displacement of each chamber by a load cell platform located below each chamber. Before the experiment, all load cell amplifiers were calibrated to a fixed chamber displacement, and the output of each amplifier was set to a gain that optimally detected freezing behavior (vernier dial $=8$; somatomotor immobility, except that required for breathing). Load-cell amplifier output $(-10$ to $+10 \mathrm{~V})$ was then digitized $(5 \mathrm{~Hz})$ and acquired online with Threshold Activity software (MED Associates). Absolute values of load-cell voltages were multiplied by 10 , which resulted in an activity score that ranged from 0 to 100 . A bout of freezing was scored if at least five contiguous loadcell values fell below the freezing threshold. In other words, activity had to be
Control

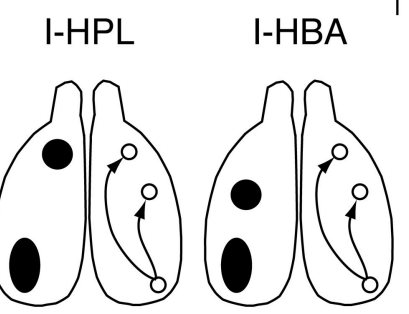

Disconnect

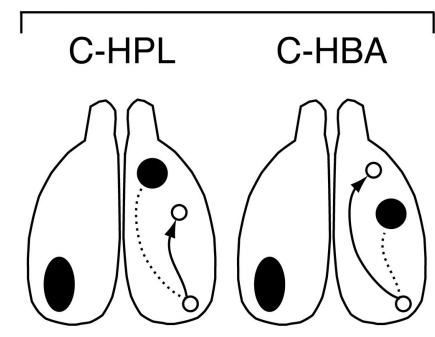

Figure 1. A schematic of the projections between the hippocampus $(H), B A$, and $P L$ in control rats and rats in which the

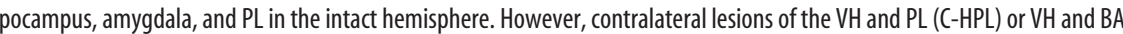
(

below threshold for at least $1 \mathrm{~s}$ to be scored as freezing behavior. This method of assessing freezing behavior correlates highly with time sampling of behavior by trained observers (Maren, 1998).

Behavioral procedures. For the immunohistochemistry experiment, 36 rats were randomly assigned to groups that were to be tested either within the extinction context (SAME) or outside the extinction context (DIFF) and those that were left in the home cages during the test session (HOME). We used a three-context $(\mathrm{ABC})$ renewal procedure in which rats were conditioned in context $\mathrm{A}$, extinguished in context $\mathrm{B}$ or $\mathrm{C}$, and then tested in context $\mathrm{C}$ (extinction and test contexts were counterbalanced). This yielded groups tested in the extinction context (SAME, ACC) or in another, familiar context that had not hosted extinction (DIFF, ABC). This renewal design is critical because it allows the assessment of fear and c-fos expression to an extinguished CS independent of fear to the context in which the CS is tested (i.e., rats are never tested in the conditioning context as they are in a typical ABA design). Moreover, all rats were tested identically and in the same physical contexts so that any differences in behavior and c-fos expression could be attributed to the meaning of the CS in that context and not the CS or context itself.

One week before all behavioral sessions, rats received unilateral intracranial injections of cholera toxin subunit b (CTb; \#104; List Biological) into the BA. The side of the injection was counterbalanced across all behavioral groups. After allowing for 1 week of recovery, rats underwent fear conditioning in context A. Conditioning consisted of five tone (CS; $10 \mathrm{~s}, 85 \mathrm{~dB}, 2 \mathrm{kHz}$ )-footshock (US; $1.0 \mathrm{~mA}, 2.0 \mathrm{~s}$ ) pairings with $60 \mathrm{~s}$ interstimulus intervals (ISIs). The chamber position of each animal was counterbalanced across each experimental group and training squad. Twenty-four hours later, animals underwent extinction in context $\mathrm{B}$ or $\mathrm{C}$ (counterbalanced across groups) in which they received 45 tone-alone $(10 \mathrm{~s})$ presentations with $30 \mathrm{~s}$ ISIs. Before this session, the rats were exposed to the alternate context (i.e., they were exposed to context B if they were extinguished in context $C$ ) to ensure that the test contexts were equally familiar for all of the rats. Twenty-four hours after extinction, the rats were returned to the observation chambers (context C) for a test session consisting of five tone-alone (10 s) presentations with $30 \mathrm{~s}$ ISIs. Rats were killed $90 \mathrm{~min}$ after the first tone presentation to assess c-fos expression induced by the test session. In all behavioral sessions, freezing was used as an index of fear.

In the disconnection experiment, 125 rats were randomly assigned to groups that would receive contralateral (C) lesions, ipsilateral (I) lesions, or sham (SH) surgeries in the VH-BA or VH-PL circuits after extinction training. Additionally, these groups were further divided into rats that would be tested outside the extinction context (DIFF) or within the extinction context (SAME). Rats were trained and extinguished, as described previously. Twenty-four to $96 \mathrm{~h}$ after extinction, the rats underwent surgery. After 1 week of recovery, the rats were placed back into either context B or context $\mathrm{C}$ for the test session [45 tone alone (10 s) presentations with a $30 \mathrm{~s}$ ISI]. In this experiment, the extinction and test contexts were counterbalanced across all groups. Renewal was assessed by measuring freezing during the first five trials of the test session in the DIFF context relative to that in the SAME context. 


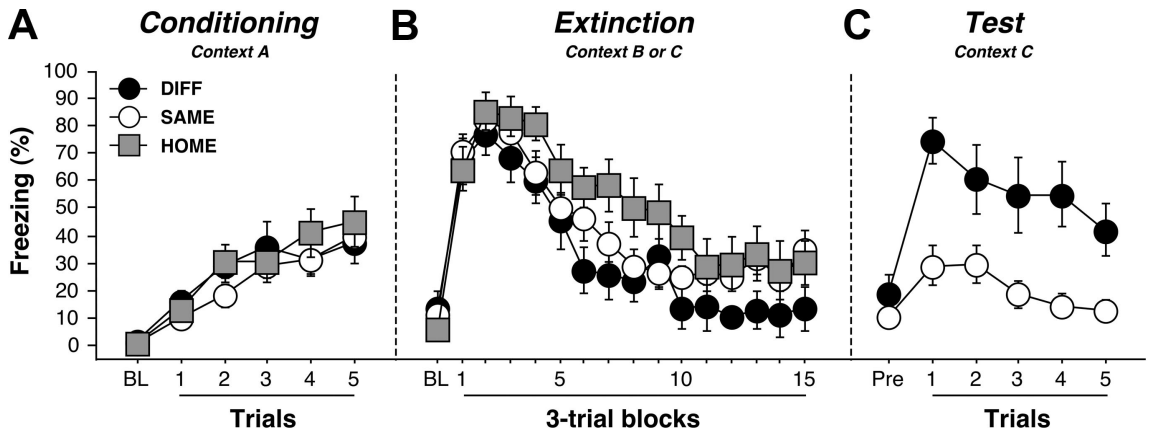

Figure 2. Conditioned freezing behavior in rats previously infused with CTb. $A$, Mean \pm SEM percentage of freezing during fear conditioning, which consisted of a 3 min baseline period followed by five tone-shock pairings. Freezing was averaged across the pre-CS baseline (BL) as well as during each of the five conditioning trials; each trial consisted of the average of freezing during each CS presentation and the subsequent ISI. $\boldsymbol{B}$, Mean \pm SEM percentage of freezing during the 45 tone-alone extinction session. Freezing was averaged across the $B L$ period as well as during the 45 extinction trials; as with conditioning, each trial consisted of the average of freezing during each CS presentation and subsequent ISI (data were binned into 15 blocks of three-trial averages). $\boldsymbol{C}$, Mean \pm SEM percentage of freezing during the test session that consisted of five tone-alone presentations with 30 s ISIs. Freezing was measured during the BL period and during the five trials, each of which consisted of a CS presentation and the subsequent ISI. Data are shown for rats that were tested outside the extinction context (DIFF; black circles), tested within the extinction context (SAME; white circles), or not tested at all (HOME; gray squares).

To assess whether the disconnections produced nonspecific impairments in freezing behavior, we ran an additional experiment with another cohort of 59 rats. The rats were conditioned, as described above, but they did not receive extinction training. Rather, they were merely exposed to either context B or context C (no CS presentations) for the same duration as the extinction session in the foregoing experiment. Twenty-four hours later, the rats received either contralateral or ipsilateral lesions placed in the $\mathrm{VH}$ and $\mathrm{BA}$ or $\mathrm{VH}$ and PL or sham surgeries. After 1 week of postoperative recovery, the rats were tested for their retention of fear to the non-extinguished CS in either context B or C. As before, the extinction and test contexts were counterbalanced across lesion groups.

Surgical procedures. Rats were anesthetized with ketamine $(100 \mathrm{mg} / \mathrm{kg}$, i.p.) and xylazine (10 mg/kg, i.p.) and given atropine sulfate $(0.4 \mathrm{mg} / \mathrm{kg}$, i.p.). After being placed into the stereotaxic apparatus (David Kopf Instruments), the scalp was incised and retracted. The head was leveled to ensure that lambda and bregma were in the same horizontal plane. In the tracing/c-fos experiment, one small burr hole was drilled to allow for a 30-gauge injector (Small Parts) to be lowered into the BA [anteroposterior (AP), $-3.1 \mathrm{~mm}$; mediolateral $(\mathrm{ML})$, $\pm 4.6-5.1 \mathrm{~mm}$; dorsoventral (DV), $-8.1 \mathrm{~mm}$ from dura]. The injector was attached to polyethylene tubing, which was connected to Hamilton syringes $(10 \mu \mathrm{l})$ located on an infusion pump. CTb (\#104; List Biological) was unilaterally infused at a rate of $0.1 \mu \mathrm{l} / \mathrm{min}$ for $1 \mathrm{~min}(0.1 \mu \mathrm{l}$ total volume; $10 \mathrm{mg} / \mathrm{ml})$. The injector remained in the BA for another 5 min to allow for the diffusion of $\mathrm{CTb}$. The hemisphere of CTb injection was counterbalanced across all behavioral groups (DIFF, SAME, and HOME).

To disconnect the $\mathrm{VH}$ and $\mathrm{BA}$ or $\mathrm{VH}$ and $\mathrm{PL}$, unilateral electrolytic lesions of these structures were placed in opposite hemispheres; ipsilateral lesions served as a control (Fig. 1). We chose to use electrolytic lesions because we were able to produce much more focal damage to the $\mathrm{BA}$ and PL than we obtained with excitotoxic lesions. For the VH-BA disconnections, insulated insect pins (size 00; except for $1 \mathrm{~mm}$ at the tip; Fine Science Tools) were placed in the VH (AP, $-6.7,-6.3,-5.8 \mathrm{~mm}$; $\mathrm{ML},+/-5.6,5.4,5.2 \mathrm{~mm}$; DV $,-5.6,-5.8,-6 \mathrm{~mm}$ from dura) and the BA (AP, $-2.85,-3.6 \mathrm{~mm}$; ML, +/ $-5 \mathrm{~mm}$; DV, $-9,-9.1 \mathrm{~mm}$ from skull). For the VH-PL disconnections, the electrodes were placed in the PL (AP, +3.6, +2.7 mm; ML, $\pm 0.5 \mathrm{~mm}$; DV, $-3.7 \mathrm{~mm}$ from skull) and

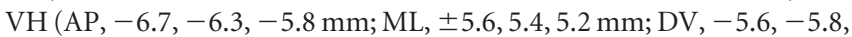
$-6 \mathrm{~mm}$ from dura). Lesions were produced by passing anodal current $(0.5 \mathrm{~mA}, 8 \mathrm{~s})$ across the insect pins. Lesion placement in the left or right hemispheres was counterbalanced. The incision was closed with stainless steel wound clips and treated with antibiotic ointment, and the animals were administered an analgesic (carprofen, $5 \mathrm{mg} / \mathrm{kg}$, i.p.). The rats recov- ered on a heating pad before returning to their home cages and were allowed 1 week for postoperative recovery.

Immunohistochemistry. Ninety minutes after the first tone presentation, rats were overdosed with pentobarbital and transcardially perfused with ice-cold $0.1 \mathrm{M} \mathrm{PBS}, \mathrm{pH} 7.4$, and $4 \%$ paraformaldehyde (PFA), $\mathrm{pH}$ 7.4. Brains were extracted and placed into 4\% PFA for $1 \mathrm{~h}$ and then transferred into a $30 \%$ sucrose $/ 0.1 \mathrm{M}$ PBS solution. Brains were mounted and cut $(30$ $\mu \mathrm{m}$ ) on a cryostat maintained at $-20^{\circ} \mathrm{C}$. Coronal sections were collected every $150 \mu \mathrm{m}$ and placed in $0.1 \mathrm{M}$ PBS with $0.2 \%$ sodium azide. Additional sections were mounted on subbed slides with $70 \%$ ethanol for Nissl staining.

Immunohistochemistry was performed on free-floating sections. Sections were first washed two times in $0.1 \mathrm{M}$ PBS for $10 \mathrm{~min}$, followed by a third wash in $0.1 \mathrm{M}$ PBS with $0.3 \%$ Triton X-100 for $10 \mathrm{~min}$. Tissue was then incubated at room temperature in 10\% normal donkey serum (NDS) and $0.3 \%$ Triton X-100 in $0.1 \mathrm{M}$ PBS for $30 \mathrm{~min}$. Sections were then immediately transferred into the primary antibody solution for a $48 \mathrm{~h}$ incubation period [goat anti-c-fos at 1:1000 (sc-52-G; Santa Cruz Biotechnology), rabbit anti-CTb at 1:3000 (C3062; Sigma), in 0.1 M PBS with 10\% NDS and $0.3 \%$ Triton $\mathrm{X}-100$ ] at $4^{\circ} \mathrm{C}$. Forty-eight hours later, tissue was washed three times in $0.3 \%$ Triton X-100 in $0.1 \mathrm{M}$ PBS for $10 \mathrm{~min}$ and then incubated in 10\% NDS for $30 \mathrm{~min}$. Sections were then incubated in the secondary antibodies [biotinylated donkey anti-goat at 1:200 (sc-2042; Santa Cruz Biotechnology), donkey anti-rabbit conjugated to Alexa Fluor 488 at 1:500 (A-21206; Invitrogen), in 0.3\% Triton X-100 and 10\% NDS in $0.1 \mathrm{M}$ PBS] for $2 \mathrm{~h}$ at room temperature. After being rinsed in $0.1 \mathrm{M} \mathrm{PBS}$ with $0.3 \%$ Triton X-100, tissue was incubated in streptavidin conjugated to Alexa Fluor 594 (1:200 in 0.3\% Triton X-100 and 10\% NDS in 0.1 M PBS; S11223; Invitrogen) for $1 \mathrm{~h}$ at room temperature. To aid in the fluorescent detection, Signal Enhancer (three drops per $8 \mathrm{ml}$ of solution; Invitrogen) was added to both the secondary antibody and streptavidin solution. After a final wash in $0.1 \mathrm{M}$ PBS, tissue was mounted onto subbed slides in $0.9 \%$ saline and coverslipped with Vectashield (H-1200; Vector Laboratories).

Image analysis. We quantified the number of CTb- and c-fos-positive nuclei in the PL, infralimbic cortex (IL), perirhinal cortex (PRh), auditory cortex (AC), and VH (consisting of the ventral subiculum and ventral CA1 subfield). Multiple images were captured for each region, consistent with other published immunohistochemical reports (Herry and Mons, 2004; Berretta et al., 2005; Kim et al., 2009). Specifically, for the prelimbic region, four images were captured (two sets bilaterally at +3.7 and $+2.7 \mathrm{~mm}$ anterior to bregma). For the infralimbic region, four images were captured (bilaterally at +3.2 and $+2.7 \mathrm{~mm}$ anterior to bregma). Three images for the $\mathrm{PRh}(-3.0,-3.6$, and $-4.0 \mathrm{~mm}$ posterior to bregma), $\mathrm{AC}(-3.6,-4.0$, and $-4.6 \mathrm{~mm}$ posterior to bregma), and $\mathrm{VH}(-5.6,-6.3$, and $-6.8 \mathrm{~mm}$ posterior to bregma) were taken on the side of the CTb injection. To verify the borders of cortical areas and the hippocampal layers, adjacent thionin-stained sections were used. All images were taken at $20 \times$ magnification $\left(443 \times 331 \mu \mathrm{m} ; 0.15 \mathrm{~mm}^{2}\right)$ with a Leica DM6000 B microscope outfitted with filters for different excitation/emission wavelengths (Hamlin et al., 2009; Knapska and Maren, 2009). For each region, the number of c-fos-positive, CTb-positive and double-labeled neurons were counted using an image analysis software program (NIH ImageJ). Importantly, double-labeled neurons were readily observable because the resulting stain was visible in a center-surround manner for c-fos and CTb, respectively (Leman et al., 2000; Marchant et al., 2009). Counts for each image of the brain region were averaged across the number of images taken, and group differences in absolute cell counts were analyzed with an ANOVA and Fisher's protected least significant difference (PLSD) post hoc tests. Results are represented as means \pm SEM. 
Histology. After behavioral testing, rats in the disconnection studies were overdosed with pentobarbital and perfused across the heart with $0.9 \%$ saline followed by $10 \%$ Formalin. Brains were extracted and postfixed in $10 \%$ Formalin for $2 \mathrm{~d}$, at which time brains were transferred into a solution of $30 \%$ sucrose in $10 \%$ Formalin. Brains were sectioned (45 $\mu \mathrm{m})$ on a cryostat maintained at $-20^{\circ} \mathrm{C}$. Tissue was wet mounted on subbed slides with $70 \%$ ethanol and subsequently stained with $0.25 \%$ thionin to verify lesion placement and extent.

Behavioral data analysis. Freezing behavior was measured continuously during all of the behavioral sessions, including the pre-CS "baseline" period as well as during tone presentations and ISI. Freezing was then analyzed and reported for each trial, which consisted of a CS presentation and the subsequent ISI. For each period, the percentage of total observations in which freezing occurred was calculated. These values were submitted to ANOVA and Fisher's PLSD post hoc tests were performed after significant omnibus $F$ ratios were obtained. All data are represented as means \pm SEM.

\section{Results}

c-fos expression in PL and VH projections to the BA after the renewal of fear

Freezing behavior during conditioning (context A), extinction (contexts $\mathrm{B}$ or $\mathrm{C}$ ), and retrieval testing (context $\mathrm{C}$ ) is displayed in Figure 2. Freezing behavior during conditioning and extinction was typical and did not differ between the groups [ $F$ values $<1$ ]. As expected, conditional freezing differed markedly between the groups during the retrieval test. Rats tested outside the extinction context (DIFF) exhibited renewal of conditional freezing to the extinguished CS, whereas those tested within the extinction context (SAME) displayed low levels of fear. This impression was confirmed in an ANOVA performed on the CS-elicited freezing across the test trials (main effect of group, $F_{(1,23)}=24.3, p<$ $0.01)$. Importantly, renewal of fear was not attributable to contextual fear because the levels of pre-CS freezing in the two contexts were not statistically different (DIFF, $19.3 \pm 6.4 \%$; SAME, $\left.10.9 \pm 2.9 \% ; F_{(1,23)}=1.9, p=0.2\right)$. Moreover, differential freezing among the SAME and DIFF groups was not attributable to physical differences in the test contexts; all testing was conducted in identical contexts with the same CS.

After retrieval testing, rats were killed to assess c-fos expression in BA afferents. A representative CTb injection site in the BA is shown in Figure 3, $A$ and $B$, along with a schematic illustration of maximal and minimal infusions (Fig. $3 C$ ). Only rats for which $\mathrm{CTb}$ labeling was confined to the BA were included in the analysis. Four rats were excluded because their CTb injections were not confined to BA. Another 12 rats were excluded because the CTb infusions were too small, resulting in weak staining. The final group sizes were DIFF $(n=9)$, SAME $(n=16)$, and HOME $(n=11)$.

Retrieval testing induced c-fos expression in the PL, IL, and $\mathrm{VH}$, and many c-fos-positive nuclei were colocalized with $\mathrm{CTb}$ positive cells (Fig. 4). The absolute counts of CTb- and c-fospositive nuclei as well as the percentage of double-labeled $[$ (double-labeled cells/total CTb cells $) \times 100]$ neurons for the PL, IL, and VH are shown in Figure 5A-C. Two-way ANOVAs with a within-subjects factor of brain region and between-subjects factor of group were performed for each dependent variable. The extent of CTb labeling in the groups did not differ in any of the brain regions (Fig. $5 A$; $p$ values $>0.05$ ), although there was a main effect of brain region $\left(F_{(4,132)}=18.6, p<0.001\right)$. Post hoc comparisons revealed that there were significantly more $\mathrm{CTb}$ positive neurons in the IL than the $\mathrm{PL}(p<0.001)$ and the $\mathrm{VH}$ $(p<0.01)$. Additionally, the AC had significantly less CTbpositive neurons than all other brain regions $(p<0.001)$, whereas the $\mathrm{PRh}$ had significantly more $\mathrm{CTb}$-positive neurons
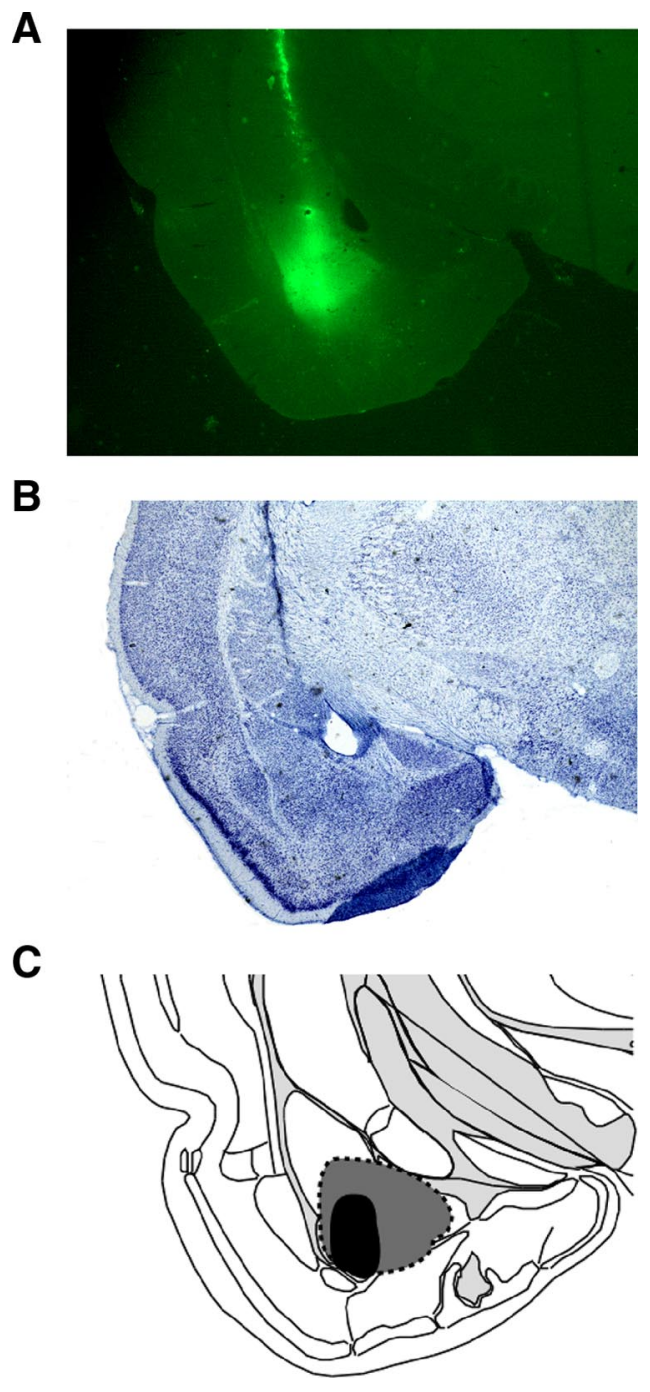

Figure 3. Illustrations of the CTb injection site and CTb spread within the BA. $A$, A representative CTb-stained coronal section displaying the site of the CTb injection. $\boldsymbol{B}$, Adjacent thionin sections were used to ensure that CTb spread did not extend beyond the boundaries of the BA. C, Schematic of CTb spread; gray indicates rats with maximal CTb spread, and black represents rats with the smallest injections of CTb. Images were adapted from Swanson (2004).

than all other brain regions $(p<0.01)$, with the exception of the IL. This pattern of retrograde labeling is generally consistent with the distribution and density of projections from these regions to the BA (Romanski and LeDoux, 1993; Pitkanen et al., 2000; Vertes, 2004).

As we have previously reported, either the renewal or suppression of fear was associated with different patterns of c-fos expression in the PL, IL, and VH (Fig. 5B). This was confirmed by significant main effects of group $\left(F_{(2,33)}=17.5, p<0.001\right)$ and brain region $\left(F_{(4,132)}=54.0, p<0.001\right)$ and a significant group $\times$ region interaction $\left(F_{(8,132)}=8.3, p<0.001\right)$ in the ANOVA. Within the PL, post hoc comparisons revealed that rats in both the SAME and DIFF groups exhibited significant increases in c-fos expression relative to rats in the HOME group $(p<0.001)$. In the IL, $c$-fos expression was greater in the SAME group relative to both the DIFF and HOME groups $(p<0.05)$; c-fos expression was not significantly different between the DIFF and HOME groups $(p>0.05)$. In the $\mathrm{VH}$, rats in the DIFF group exhibited significantly more $\mathrm{c}$-fos expression than rats in the HOME group $(p<0.05)$. There were no significant group differences for $c$-fos 


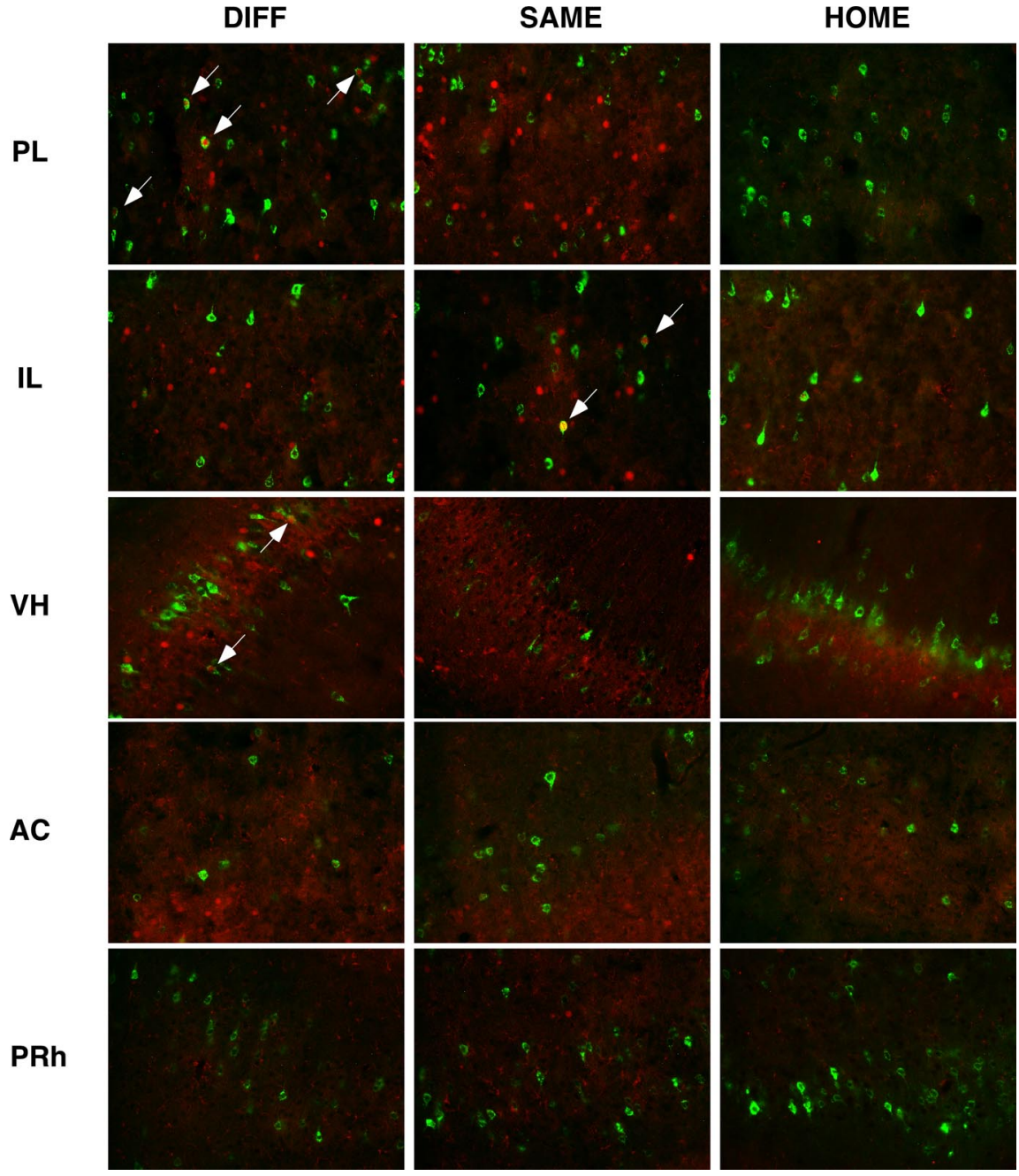

Figure 4. Photomicrographs of representative double-labeled neurons in the PL, IL, VH, AC, and PRh for each behavioral group (DIFF, SAME, HOME). White arrows indicate double-labeled neurons; CTb-positive neurons are stained green, and c-fos-positive neurons are stained red.

expression in the AC and PRh $(p>0.05)$. This suggests that the $\mathrm{PL}, \mathrm{IL}$, and VH are differentially engaged by the renewal or suppression of fear to an extinguished CS.

Of considerable interest is the proportion of retrogradely labeled neurons in each area that express c-fos during retrieval testing (Fig. 5C). We found significant group differences in the number of double-labeled neurons in the PL, IL, and VH. In particular, the VH and PL exhibited significantly more doublelabeled neurons in the DIFF condition relative to the SAME and HOME conditions, whereas the number of double-labeled cells in the IL was greatest in the SAME condition. These impressions were confirmed in the ANOVA by significant main effects of group $\left(F_{(2,32)}=9.4, p<0.001\right)$ and brain region $\left(F_{(4,128)}=22.2\right.$, $p<0.001)$ as well as a group $\times$ brain region interaction $\left(F_{(8,128)}=\right.$ $8.1, p<0.001)$. Within the PL, post hoc comparisons revealed that there were significantly more double-labeled neurons in the DIFF group than the SAME $(p<0.001)$ and the HOME $(p<0.001)$ groups. Within the IL, however, there were significantly more double-labeled neurons in the SAME group relative to the DIFF $(p<0.05)$ and HOME $(p<0.001)$ groups. Additionally, there were more double-labeled neurons in the IL in the DIFF group than the HOME group $(p<0.05)$. In the $\mathrm{VH}$, there were significantly more double-labeled neurons in the DIFF group relative to SAME $(p<0.01)$ and HOME $(p<$ $0.005)$ groups. Within the $\mathrm{VH}$, the SAME and HOME groups were not significantly different from one another, and there were no significant group differences in the AC or PRh. These data reveal that BAprojecting neurons in the $\mathrm{VH}$ and $\mathrm{PL}$ are preferentially engaged during fear renewal, whereas those in IL are engaged by the suppression of fear during the expression of extinction. Importantly, these patterns of c-fos expression are related not to the specific sensory properties of the CS or the test context (both of which are the same for the SAME and DIFF groups) but rather the meaning of the CS in a particular context.

Interestingly, the proportion of doublelabeled neurons across the PL, IL, and VH within a retrieval condition was significantly different. Within the DIFF condition, post hoc comparisons showed that the PL had the highest proportion of double-labeled neurons relative to all of the other brain regions $(p<0.05)$. Hence, although BA-projecting neurons in the $\mathrm{VH}$ exhibited greater $\mathrm{c}$-fos expression in the DIFF relative to SAME conditions, the proportion of BA-projecting neurons exhibiting $\mathrm{c}$-fos was much lower than that in the PL. In the SAME condition, however, the IL exhibited the highest proportion of double-labeled cells relative to the other groups $(p<0.05)$. Together, these results indicate that a greater number of BAprojecting neurons in the PL are engaged during renewal than BA-projecting neurons in the VH. Conversely, a greater number of BA-projecting neurons in the IL are engaged during the retrieval of extinction relative to the $\mathrm{PL}$ and $\mathrm{VH}$.

\section{Disruption of direct or indirect hippocampal projections to} the BA impairs fear renewal

To determine the role of direct and indirect projections of the $\mathrm{VH}$ to the BA in the renewal of fear, we disconnected the $\mathrm{VH}$ and $\mathrm{BA}$ or $\mathrm{VH}$ and $\mathrm{PL}$ with asymmetric unilateral lesions in each structure (Fig. 1). Control rats received the same lesions, but they were localized in the same hemisphere, leaving connections between the VA, PL, and BA intact in the opposite hemisphere. Representative photomicrographs of the lesions are shown in Figure 6. We used strict criteria when analyzing the histology to ensure that only animals with focal lesions in the targeted brain regions were included in the analysis. For the BA, we excluded animals for which the lesions extended dorsally into the lateral amygdala. Similarly, subjects were excluded from the analysis if lesions of the PL encroached on either the cingulate cortex dorsally or the IL ventrally. Finally, we required that $\mathrm{VH}$ lesions were localized to the ventral subiculum and ventral CA1 area. If damage to the entorhinal cortex or dentate gyrus was apparent, then the subject was excluded from the analyses. In all cases, lesions had to encompass at least two-thirds of the intended target to be considered a "hit" and included in the statistical analyses. Based on these criteria, we excluded 68 rats, which left a total of 125 animals in 
the analysis. The final group sizes were as follows: SH-SAME, $n=20$; SH-DIFF, $n=$ 22; I-HPL-SAME, $n=8$; I-HPL-DIFF, $n=6$; I-HBA-SAME, $n=14$; I-HBADIFF, $n=14$; C-HPL-SAME, $n=8$; C-HPL-DIFF, $n=8$; C-HBA-SAME, $n=$ 12; C-HBA-DIFF, $n=13$. It is important to note that the relatively large number of rats in the sham groups is a consequence of the exclusion of many rats with lesions that did not meet our histological criteria for inclusion in the analysis.

Freezing behavior during the conditioning, extinction, and test session is displayed in Figure 7. Before surgery, there were no differences in freezing behavior during the conditioning and extinction sessions $(p$ values $>0.1$ ). During the test session, pre-CS freezing in the SAME and DIFF conditions for each group did not differ from one another $\left(F_{(1,115)}=2.4\right.$, $p=0.1)$, nor did pre-CS freezing in any of groups differ from one another $\left(F_{(4,115)}=\right.$ $0.9, p=0.4$; data not shown). We therefore normalized the average CS-elicited freezing (freezing during the CS and ISIs averaged across each test trial) to the pre-CS baseline for each rat. As shown in Figure 7, the groups exhibited marked differences in the degree of fear renewal during the test session. This was indicated by a main effect of lesion $\left(F_{(4,115)}=5.2, p<\right.$ $0.001)$ and significant interaction of lesion and test context in the $\operatorname{ANOVA}\left(F_{(4,115)}=\right.$ $2.9, p<0.05)$. Post hoc comparisons revealed that both sham animals and rats with ipsilateral lesions (I-HPL and I-HBA) renewed their fear to the CS outside the extinction context ( $p$ values $<0.05$ ). However, rats with contralateral lesions (C-HPL and C-HBA) failed to exhibit renewal ( $p$ values $>0.05$ ). Hence, either VH-BA or VH-PL disconnections eliminated the contextual retrieval of fear after extinction.

Although control rats with ipsilateral lesions exhibited normal renewal, it is possible that the deficit in rats with contralateral disconnections was attributable to a nonspecific impairment in fear expression to the auditory CS rather than a deficit in renewal per se. To examine this possibility, we conducted an additional experiment examining the consequences of ipsilateral or contralateral lesions in the VH-BA and VH-PL circuits on the expression of conditioned fear to an auditory CS that was not extinguished. The behavioral paradigm was identical to the previous experiment, except that animals did not hear auditory CSs during the postconditioning session in context B (i.e., there was no extinction training). Twenty-one rats were excluded from the analysis because their lesions did not meet our histological criteria; this left a total of 59 rats in the analyses. The final group sizes were as follows: $\mathrm{SH}, n=15$; I-HPL, $n=8$; I-HBA, $n=14$; C-HPL, $n=7$; C-HBA, $n=15$. Freezing behavior during fear conditioning and the context exposure session was not different between any of the groups ( $p$ values $>0.05$ ). As shown in Figure 8, the test session reveals that neither VH-BA nor VH-PL disconnections affected the expression of freezing behavior during the retention test $\left(F_{(4,54)}=1.8, p>0.1\right)$. Nonetheless, there was a nonsignificant trend for reduced freezing in all of the rats with lesions, regardless of whether they were placed ipsilaterally or contralaterally in the VH-BA and VH-PL circuits. However, this general suppression of fear in rats with ipsilateral or contralateral lesions does not account for the selective deficit in renewal in rats with contralateral lesions in the previous experiment. Therefore, deficits in fear renewal in rats with contralateral disconnections in the previous experiment are not merely attributable to deficits in the expression of conditional freezing.

\section{Discussion}

The present data reveal that convergent input from both the $\mathrm{VH}$ and PL to the BA is required for the renewal of fear after extinction. First, we found that significantly more BA-projecting neurons in the PL and $\mathrm{VH}$ exhibited c-fos expression during the renewal of fear compared with the recall of extinction. Conversely, more BA-projecting neurons within the IL exhibited c-fos expression during the recall of extinction than during renewal. Second, we showed that disconnection of either the direct projection from $\mathrm{VH}$ to $\mathrm{BA}$ or indirect projection via the PL eliminated the renewal of fear. These results reveal that the contextual regulation of extinguished fear memories requires a distributed neural network involving the $\mathrm{VH}, \mathrm{PL}$, and BA.

These results replicate and extend recent work from our laboratory that has shown that c-fos expression in the prefrontal cortex and hippocampus is regulated by the context in which an extinguished CS is retrieved (Knapska and Maren, 2009). In both studies, for example, we found that c-fos expression in the IL was signifi- 
cantly greater during extinction recall than during renewal. The present results extend this work by demonstrating that BAprojecting neurons within the IL are strongly recruited during extinction recall. Interestingly, we also found that BA-projecting neurons in the IL are engaged during renewal of fear, albeit to a lesser extent than those in the PL. Although IL activation in the renewal context was weak, it suggests that IL neurons that project to the BA may nonetheless have some role in promoting fear expression during renewal. Collectively, these data support the view that the IL has an important role in the retrieval of extinction memories (Milad and Quirk, 2002; Sierra-Mercado et al., 2006).

The present results also confirm our previous findings showing greater c-fos expression in the VH and PL during renewal of fear to an extinguished CS (Knapska and Maren, 2009). However, unlike Knapska and Maren (2009), we observed an increase in c-fos expression within the PL during both the renewal of fear and during the expression of extinction (at levels similar to that in the IL). One potential reason for this discrepancy may be the differences in renewal paradigms used in each study; we used a three-context renewal design (ABC), whereas Knapska and Maren (2009) used a twocontext design (AAB). In addition, different immunohistochemical detection methods for $\mathrm{c}$-fos were used in each study. Regardless of these differences, however, both studies clearly demonstrate that neurons within $\mathrm{VH}$ and PL are engaged during fear renewal.

Of course, it is possible that the retrograde labeling we observed in BA afferents is attributable to CTb spread into neighboring amygdala regions. For example, CTb labeling in the IL could be a result of $\mathrm{CTb}$ diffusion into the adjacent intercalated cell masses (ITC), clusters of GABAergic interneurons that receive glutamatergic projections from the IL (Berretta et al., 2005). We believe that this possibility is unlikely, however, insofar as we were careful to only include subjects for which we had focal BA $\mathrm{CTb}$ injections. In addition, the afferent pathways we chose to investigate have very specific connections with the BA. For example, the BA is the only region of the amygdala that receives robust input from the ventral CA1 area of the hippocampus (Pitkanen et al., 2000). Given that cholera toxin is a selective monosynaptic retrograde tracer (Bruce and Grofova, 1992; Conte et al., 2009), we are confident that the CTb labeling we have observed reflects specific afferent projections of the BA.

Although considerable data indicate an important role for the hippocampus in the renewal of extinguished fear (Corcoran and Maren, 2001; Corcoran et al., 2005; Hobin et al., 2006; Maren, 2011), we now show for the first time that the PL has an important role in this process. We found significantly greater numbers of BAprojecting neurons in the $\mathrm{PL}$ relative to the $\mathrm{VH}$ during the renewal of fear, and disconnection of projections from the VH to PL eliminated fear renewal. The involvement of the PL in renewal is consistent with the emerging view that it has an important role in the expression of conditioned fear. For example, previous work has shown that CSevoked neuronal activity in the PL parallels freezing behavior during conditioning and extinction (Burgos-Robles et al., 2009), and inactivation of the PL disrupts the expression of learned fear (Corcoran and Quirk, 2007; Sierra-Mercado et al., 2011). It should be noted that the PL is not required for fear expression because PL inactivation spares unconditioned freezing (Corcoran and Quirk, 2007). Moreover, we have found that the expression of fear to a nonextinguished CS does not induce c-fos in PL neurons (Knapska and Maren, 2009). These data suggest that the PL, like the hippocampus, has an important role in contextual memory retrieval rather than in fear expression per se.

Because both the VH and PL are important routes by which contextual information reaches the BA to regulate fear output, we
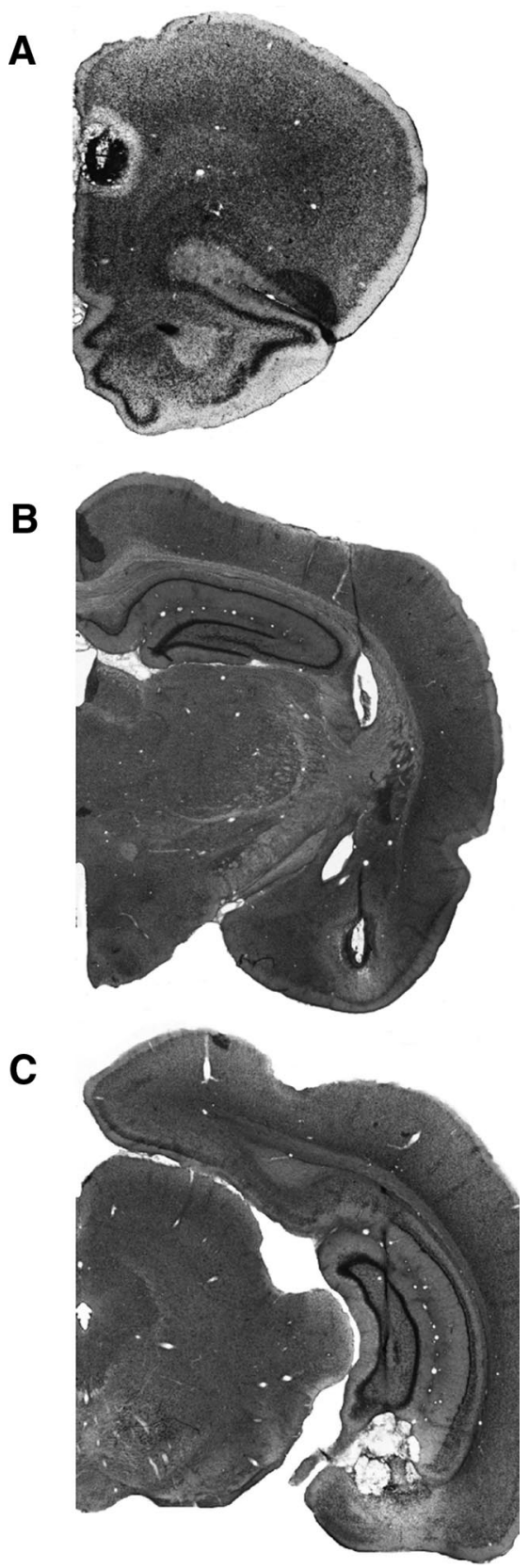

Figure 6. Photomicrographs of thionin-stained coronal sections showing representative lesions in the $\mathrm{PL}(\boldsymbol{A}), \mathrm{BA}(\boldsymbol{B})$, and $\mathrm{VH}(\boldsymbol{C})$ for three different rats in the disconnection experiment.

propose that the convergence of hippocampal and prefrontal input in the BA is essential for the contextual regulation of extinction memory (Fig. 9). In support of this model, interrupting hippocampal input to either the BA or PL eliminates the renewal of fear. In the former case, $\mathrm{VH}$ and $\mathrm{BA}$ disconnections spare $\mathrm{BA}-\mathrm{PL}$ interconnection in one hemisphere but isolate this circuit from hippocampal input. In the latter case, $\mathrm{VH}$ and $\mathrm{PL}$ disconnections spare $\mathrm{VH}-\mathrm{BA}$ connections in one hemisphere but isolate this circuit from prelimbic input. By this view, convergent excitatory input from the $\mathrm{VH}$ and PL may be required to overcome inhibitory networks in the amygdala that suppress fear responses after extinction. Extinctionrelated inhibition might come from either local inhibitory interneurons in BA (Chhatwal et al., 2005) or through IL-gated inhibitory networks in ITC clusters (Paré et al., 2004; Amano et al., 2010). Although neurons in the BA that respond to CSs during fear renewal 
receive hippocampal input, it is not clear that they also receive convergent prelimbic input (Herry et al., 2008). However, it has been reported that single $\mathrm{VH}$ neurons project to both PL and BA (Ishikawa and Nakamura, 2006). This raises the possibility that $\mathrm{VH}$ neurons projecting to $\mathrm{PL}$ and $\mathrm{BA}$ form a common anatomical hub to regulate excitability in both the PL and BA, as well as encouraging coherence in PL-BA activity (Seidenbecher et al., 2003; Pape et al., 2005; Adhikari et al., 2010). Nonetheless, our data do not address whether single BA neurons receive convergent $\mathrm{VH}$ and $\mathrm{PL}$ input or whether $\mathrm{VH}$ and $\mathrm{PL}$ projections that converge on the BA do so on different populations of BA neurons. Dual anterograde tracing of $\mathrm{VH}$ and $\mathrm{PL}$ projections to the amygdala, as well as simultaneous neural recordings in this network, will be important steps to assess this anatomical question as well as the validity of this model.

Another possibility is that the convergence of $\mathrm{VH}$ and $\mathrm{BA}$ information in the $\mathrm{PL}$, rather than $\mathrm{VH}-\mathrm{PL}$ convergence in $\mathrm{BA}$, is required for fear renewal. It is well known, for example, that the BA has robust projections to both the medial prefrontal cortex and hippocampus (Pitkanen et al., 2000; Hoover and Vertes, 2007) and that BA inactivation impairs fear renewal (Herry et al., 2008). It is also apparent from our circuit model (Fig. 9) that $\mathrm{VH}-\mathrm{BA}$ and VH-PL disconnections eliminate not only $\mathrm{VH}$ and $\mathrm{PL}$ convergence in $\mathrm{BA}$ but also the convergence of $\mathrm{VH}$ and $\mathrm{BA}$ input in PL. Consistent with the possibility that BA and $\mathrm{VH}$ convergence in PL is important for fear renewal, Herry et al. (2008) report that neurons in the BA that are active during fear renewal project to the medial prefrontal cortex. Indeed, PL neurons receive convergent hippocampal and amygdala input (Ishikawa and Nakamura, 2003). Hence, this alternative model predicts that BA inactivation, which impairs fear renewal (Herry et al., 2008), would also eliminate renewalinduced c-fos signals in the PL. Experiments to test this hypothesis are underway.

In conclusion, our results provide new insight into the neural circuitry involved in the contextual regulation of fear memories after extinction. Specifically, we found that BA-projecting neurons in both the PL and VH are preferentially active during the renewal of fear to an extinguished CS. Moreover, disconnection of either the direct or indirect routes by which the $\mathrm{VH}$ projects to the $\mathrm{BA}$ impaired the renewal of fear memories after extinction. Because fear renewal poses a challenge to patients and clinicians, it is critical to understand how dysfunction in hippocampal-prefrontal-amygdaloid connectivity underlies psychopathology. Insights into the neural mechanisms of fear and extinction promise to facilitate the development of more effective therapeutic interventions for individuals suffering from fear and anxiety disorders, such as posttraumatic stress disorder.
A

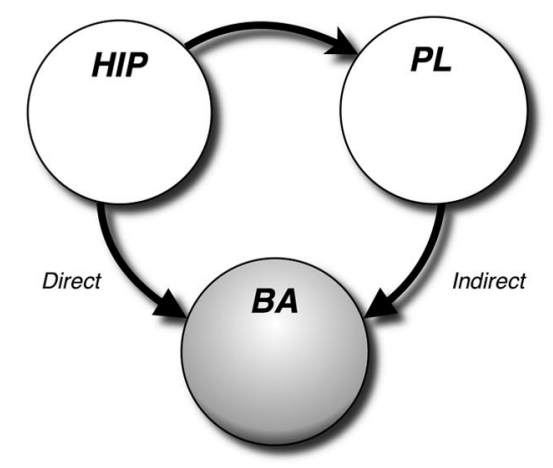

B

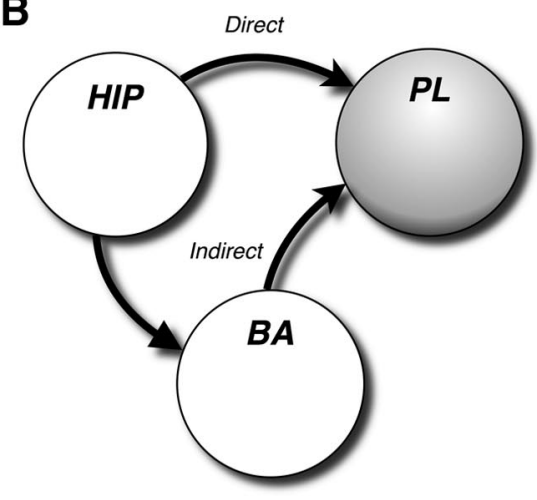

Figure 9. Circuit model of hippocampal-prefrontal-amygdaloid interactions in the renewal of fear. $\boldsymbol{A}$, In this scenario, both direct and indirect projections of the VH to the BA are required for the renewal of fear after extinction. Disconnection of either the direct or indirect pathways deprives the BA of convergent input from the VH and PL. B, Another possibility is that convergence of direct and indirect projections from the VH to the PL mediate the renewal of fear. Indeed, disconnection of either the VH-PL or VH-BA projection prevents convergence of VH and BA input in the PL. 


\section{References}

Adhikari A, Topiwala MA, Gordon JA (2010) Synchronized activity between the ventral hippocampus and the medial prefrontal cortex during anxiety. Neuron 65:257-269.

Amano T, Unal CT, Paré D (2010) Synaptic correlates of fear extinction in the amygdala. Nat Neurosci 13:489-494.

Berretta S, Pantazopoulos H, Caldera M, Pantazopoulos P, Paré D (2005) Infralimbic cortex activation increases c-Fos expression in intercalated neurons of the amygdala. Neuroscience 132:943-953.

Blum S, Hebert AE, Dash PK (2006) A role for the prefrontal cortex in recall of recent and remote memories. Neuroreport 17:341-344.

Bouton ME, Bolles RC (1979) Role of conditioned contextual stimuli in reinstatement of extinguished fear. J Exp Psychol Anim Behav Process 5:368-378.

Bruce K, Grofova I (1992) Notes on a light and electron microscopic double-labeling method combining anterograde tracing with Phaseolus vulgaris leucoagglutinin and retrograde tracing with cholera toxin subunit b. J Neurosci Methods 45:23-33.

Burgos-Robles A, Vidal-Gonzalez I, Quirk GJ (2009) Sustained conditioned responses in prelimbic prefrontal neurons are correlated with fear expression and extinction failure. J Neurosci 29:8474-8482.

Canteras NS, Swanson LW (1992) Projections of the ventral subiculum to the amygdala, septum, and hypothalamus: a PHAL anterograde tracttracing study in the rat. J Comp Neurol 324:180-194.

Chhatwal JP, Myers KM, Ressler KJ, Davis M (2005) Regulation of gephyrin and GABAA receptor binding within the amygdala after fear acquisition and extinction. J Neurosci 25:502-506.

Conte WL, Kamishina H, Reep RL (2009) The efficacy of the fluorescent conjugates of cholera toxin subunit B for multiple retrograde tract tracing in the central nervous system. Brain Struct Funct 213:367-373.

Corcoran KA, Maren S (2001) Hippocampal inactivation disrupts contextual retrieval of fear memory after extinction. J Neurosci 21:1720-1726.

Corcoran KA, Quirk GJ (2007) Activity in prelimbic cortex is necessary for the expression of learned, but not innate, fears. J Neurosci 27:840-844.

Corcoran KA, Desmond TJ, Frey KA, Maren S (2005) Hippocampal inactivation disrupts the acquisition and contextual encoding of fear extinction. J Neurosci 25:8978-8987.

Fanselow MS (2000) Contextual fear, gestalt memories, and the hippocampus. Behav Brain Res 110:73-81.

Hamlin AS, Clemens KJ, Choi EA, McNally GP (2009) Paraventricular thalamus mediates context-induced reinstatement (renewal) of extinguished reward seeking. Eur J Neurosci 29:802-812.

Herry C, Mons N (2004) Resistance to extinction is associated with impaired immediate early gene induction in medial prefrontal cortex and amygdala. Eur J Neurosci 20:781-790.

Herry C, Ciocchi S, Senn V, Demmou L, Müller C, Lüthi A (2008) Switching on and off fear by distinct neuronal circuits. Nature 454:600-606.

Hobin JA, Goosens KA, Maren S (2003) Context-dependent neuronal activity in the lateral amygdala represents fear memories after extinction. J Neurosci 23:8410-8416.

Hobin JA, Ji J, Maren S (2006) Ventral hippocampal muscimol disrupts context-specific fear memory retrieval after extinction in rats. Hippocampus 16:174-182.

Hoover WB, Vertes RP (2007) Anatomical analysis of afferent projections to the medial prefrontal cortex in the rat. Brain Struct Funct 212:149-179.

Ishikawa A, Nakamura S (2003) Convergence and interaction of hippocampal and amygdalar projections within the prefrontal cortex in the rat. J Neurosci 23:9987-9995.

Ishikawa A, Nakamura S (2006) Ventral hippocampal neurons project axons simultaneously to the medial prefrontal cortex and amygdala in the rat. J Neurophysiol 96:2134-2138.

Kim JH, Hamlin AS, Richardson R (2009) Fear extinction across development: the involvement of the medial prefrontal cortex as assessed by temporary inactivation and immunihistochemistry. J Neurosci 29:10802-10808.
Knapska E, Maren S (2009) Reciprocal patterns of c-Fos expression in the medial prefrontal cortex and amygdala after extinction and renewal of conditioned fear. Learn Mem 16:486-493.

Leman S, Viltart O, Sequeira H (2000) Double immunocytochemistry for the detection of Fos protein in retrogradely identified neurons using cholera toxin B subunit. Brain Res Brain Res Protoc 5:298-304.

Likhtik E, Pelletier JG, Paz R, Paré D (2005) Prefrontal control of the amygdala. J Neurosci 25:7429-7437.

Marchant NJ, Hamlin AS, McNally GP (2009) Lateral hypothalamus is required for context-induced reinstatement of extinguished reward seeking. J Neurosci 29:1331-1342.

Maren S (1998) Overtraining does not mitigate contextual fear conditioning deficits produced by neurotoxic lesions of the basolateral amygdala. J Neurosci 18:3088-3097.

Maren S (2001) Neurobiology of Pavlovian fear conditioning. Annu Rev Neurosci 24:897-931.

Maren S (2005) Building and burying fear memories in the brain. Neuroscientist 11:89-99.

Maren S (2011) Seeking a spotless mind: extinction, deconsolidation, and erasure of fear memory. Neuron 70:830-845.

Maren S, Hobin JA (2007) Hippocampal regulation of context-dependent neuronal activity in the lateral amygdala. Learn Mem 14:318-324.

Mcdonald AJ, Mascagni F, Guo L (1996) Projections of the medial and lateral prefrontal cortices to the amygdala: a Phaseolus vulgaris leucoagglutinin study in the rat. Neuroscience 71:55-75.

Milad MR, Quirk GJ (2002) Neurons in medial prefrontal cortex signal memory for fear extinction. Nature 420:70-74.

Olton DS, Walker JA, Wolf WA (1982) A disconnection analysis of hippocampal function. Brain Res 233:241-253.

Pape HC, Narayanan RT, Smid J, Stork O, Seidenbecher T (2005) Theta activity in neurons and networks of the amygdala related to long-term fear memory. Hippocampus 15:874-880.

Paré D, Quirk GJ, Ledoux JE (2004) New vistas on amygdala networks in conditioned fear. J Neurophysiol 92:1-9.

Pitkänen A, Pikkarainen M, Nurminen N, Ylinen A (2000) Reciprocal connections between the amygdala and the hippocampal formation, perirhinal cortex, and postrhinal cortex in rat. A review. Ann N Y Acad Sci 911:369-391.

Quirk GJ, Mueller D (2008) Neural mechanisms of extinction learning and retrieval. Neuropsychopharmacology 33:56-72.

Romanski LM, LeDoux JE (1993) Information cascade from primary auditory cortex to the amygdala: corticocortical and corticoamygdaloid projections of temporal cortex in the rat. Cereb Cortex 3:515-532.

Seidenbecher T, Laxmi TR, Stork O, Pape HC (2003) Amygdalar and hippocampal theta rhythm synchronization during fear memory retrieval. Science 301:846-850.

Sierra-Mercado D Jr, Corcoran KA, Lebrón-Milad K, Quirk GJ (2006) Inactivation of the ventromedial prefrontal cortex reduces expression of conditioned fear and impairs subsequent recall of extinction. Eur J Neurosci 24:1751-1758.

Sierra-Mercado D, Padilla-Coreano N, Quirk GJ (2011) Dissociable roles of prelimbic and infralimbic cortices, ventral hippocampus, and basolateral amygdala in the expression and extinction of conditioned fear. Neuropsychopharmacology 36:529-538.

Swanson LW (2004) Brain maps: structure of the rat brain, Ed 3. San Diego: Elsevier Academic.

Vertes RP (2004) Differential projections of the infralimbic and prelimbic cortex in the rat. Synapse 51:32-58.

Vertes RP (2006) Interactions among the medial prefrontal cortex, hippocampus and midline thalamus in emotional and cognitive processing in the rat. Neuroscience 142:1-20.

Vidal-Gonzalez I, Vidal-Gonzalez B, Rauch SL, Quirk GJ (2006) Microstimulation reveals opposing influences of prelimbic and infralimbic cortex on the expression of conditioned fear. Learn Mem 13:728-733. 